\title{
BMJ Open Integrating community health volunteers into non-communicable disease management among Syrian refugees in Jordan: a causal loop analysis
}

\author{
Parveen K Parmar (10 , ${ }^{1}$ Fatma Rawashdah, ${ }^{2}$ Nahla Al-Ali, ${ }^{3}$ Raeda Abu Al Rub, ${ }^{3}$ \\ Muhammad Fawad, ${ }^{2}$ Khaldoun Al Amire, ${ }^{2}$ Rowaida Al-Maaitah, ${ }^{3}$ \\ Ruwan Ratnayake (i) 4,5
}

To cite: Parmar PK, Rawashdah F, Al-Ali N, et al. Integrating community health volunteers into noncommunicable disease management among Syrian refugees in Jordan: a causal loop analysis. BMJ Open 2021;11:e045455. doi:10.1136/ bmjopen-2020-045455

- Prepublication history for this paper is available online. To view these files, please visit the journal online (http://dx.doi. org/10.1136/bmjopen-2020045455).

Received 09 0ctober 2020 Revised 28 March 2021 Accepted 29 March 2021

Check for updates

(c) Author(s) (or their employer(s)) 2021. Re-use permitted under CC BY-NC. No commercial re-use. See rights and permissions. Published by BMJ.

For numbered affiliations see end of article.

Correspondence to Dr Parveen K Parmar; pparmar@usc.edu

\section{ABSTRACT}

Objectives Globally, there is emerging evidence on the use of community health workers and volunteers in lowincome and middle-income settings for the management of non-communicable diseases (NCDs), provision of out-of-clinic screening, linkage with health services, promotion of adherence, and counselling on lifestyle and dietary changes. Little guidance exists on the role of this workforce in supporting NCD care for refugees who lack access to continuous care in their host country. The goals of this work were to evaluate the current roles of community health volunteers (CHVs) in the management of diabetes and hypertension (HTN) among Syrian refugees and to suggest improvements to the current primary care model using community health strategies.

Setting and participants A participatory, multistakeholder causal loop analysis workshop with representatives from the Ministry of Health of Jordan, non-governmental organisations, United Nations agencies, CHVs and refugee patients was conducted in June 2019 in Amman, Jordan.

Primary outcome This causal loop analysis workshop was used to collaboratively develop a causal loop diagram and CHV strategies designed to improve the health of Syrian refugees with diabetes and HTN living in Jordan. Results During the causal loop analysis workshop, participants collaboratively identified and mapped how CHVs might improve care among diagnosed patients. Possibilities identified included the following: providing psychosocial support and foundational education on their conditions, strengthening self-management of complications (eg, foot checks), and monitoring patients for adherence to medications and collection of basic health monitoring data. Elderly refugees with restricted mobility and/or uncontrolled disease were identified as a key population where CHVs could provide home-based blood glucose and blood pressure measurement and targeted health education to provide more precise monitoring. Conclusions CHV programmes were cited as a key strategy to implement secondary prevention of morbidity and mortality among Syrian refugees, particularly those at high risk of decompensation.

\section{Strengths and limitations of this study}

- The findings of this study resulted from convening of a group of diverse stakeholders related to diabetes and high blood pressure among Syrian refugees in Jordan.

- This study provides concrete ways in which a community health volunteer programme might support Syrian refugees in Jordan with diabetes and hypertension.

- The findings of this study may have been limited by translations errors or a lack of ability to express views openly in a mixed stakeholder setting.

\section{INTRODUCTION}

More than fivemillion Syrian refugees are displaced throughout the Middle East as a result of the conflict in Syria, with 655000 displaced in Jordan. ${ }^{1}$ The majority of these refugees in Jordan live outside of camps. ${ }^{2}$ Among non-camp Syrian refugees $\geq 18$ years of age living in Ramtha and Mafraq governorates in Jordan, 9.8\%, $17.2 \%$ and $7.3 \%$ reported diagnoses of diabetes, hypertension (HTN) or both conditions. ${ }^{3}$ Among Syrian refugees $\geq 30$ years of age, $22.9 \%$ of those with above-threshold random blood sugar and $39.1 \%$ with above-threshold blood pressure did not report a previous diagnosis. ${ }^{3}$ Refugees have been displaced for many years and may have developed these conditions after leaving Syria. ${ }^{4}$ Prolonged displacement and a high burden of non-communicable diseases (NCDs) require that primary care predominantly delivered by non-governmental organisations (NGOs) be optimised to prevent severe morbidity and mortality. ${ }^{5-9}$

Syrian refugees in Jordan access healthcare in fragmented ways. In camp settings, 
NGOs typically provide clinical care through a primary care model that favours curative therapies over preventive care.$^{10}$ Syrian refugees in periurban and urban areas access care via a patchwork of multiple health providers. These include NGO facilities that usually provide primary care services and medications at low or no cost, Ministry of Health $(\mathrm{MoH})$ clinics that provide services for low cost but often have limited resources and private clinics which typically provide more expensive services. While NGO clinics often provide free services, referrals to secondary and specialist care, diagnostics and laboratories typically require out-of-pocket payments. ${ }^{11}{ }^{12}$ Essential medications may have limited availability due to funding issues and stock-outs at both NGO and $\mathrm{MoH}$ facilities. Increasingly, NGOs use cohorts to provide more comprehensive services to patients with NCDs in Jordan, which create challenges for those who find themselves excluded from these systems. ${ }^{8}$ Changing rules governing refugee access to $\mathrm{MoH}$ clinics have resulted in further confusion and barriers in accessing care. ${ }^{11}$

Studies have shown positive impact of community health workers on NCD management in low-income and middle-income settings. ${ }^{13-18}$ However, limited guidance exists on the specific role community health volunteers (CHVs) might play in delivering care to refugees living with NCDs in protracted displacement settings. Since 2011, the International Rescue Committee (IRC) has administered Jordan's largest CHV programme, serving both Syrian refugees in non-camp settings as well as uninsured Jordanian patients. CHVs are refugees or Jordanians who may or may not have prior health experience. They receive an honorarium to volunteer a limited number of days per month. CHVs provide basic health education and referrals to primary care at IRC clinics when health issues are identified. This programme historically supported primary care functions for all household members, including monitoring, referral and health education for child health, prenatal and antenatal care, and other health conditions. The specific role that CHVs might play in NCD care, from linkage to care, out-of-clinic monitoring, or support of medication adherence to early screening for complications or early disease requires careful consideration from multiple stakeholders.

Causal loop analysis, drawn from system dynamics theory, provides a means to understand complex systems and, when done in a participatory manner, suggests programming that is sensitive to the strengths and constraints of health systems. ${ }^{19-21}$ Healthcare for refugees can be considered a complex adaptive system which results from the interactions of multiple actors (eg, beneficiaries, NGOs and health workers) and processes (eg, registration, diagnosis and adherence to care). Interactions between various components of this type of system are non-linear and difficult to predict. Causal loop analysis provides a means to qualitatively understand complex systems, and potential consequences of modifications and new programming within the system. ${ }^{19} 2223$
A participatory causal loop analysis workshop was convened in June 2019 to explore the existing models for CHV programmes in Jordan and the means by which these programmes might be improved to fill in gaps for the provision of continuous healthcare to Syrian refugees with diabetes and HTN.

\section{METHODS}

The researchers co-developed the preliminary causal loop diagram (CLD) in order to provide a platform for stakeholders to improve upon via a multiphase group model building exercise. In the first phase, a preliminary CLD was developed by the research team, after discussions with IRC health staff and document review. PKP (co-principal investigator) and FR (research manager), both female, conducted 20 key informant interviews (KIIs) in May-June 2019 in Irbid and Mafraq governorates. PKP is an experienced mixed-methods researcher and FR is Arabic-speaking and has expertise in community health, nursing and research.

Key informants were purposively selected to include experts in NCDs from the $\mathrm{MoH}$, focal points of national and international health NGOs, and IRC programmes. Additionally, CHVs, Syrian patients with diabetes and HTN, and healthcare providers (medical doctors, registered nurses and administrators) were included. The initial list of key informants was created in collaboration with IRC staff, and this list was iteratively grown by asking interviewees to recommend relevant others (box 1). The majority of individuals interviewed had previously interacted with the researchers in the context of the broader research project on NCDs among Syrian refugees in

\section{Box 1}

\section{Individuals interviewed}

- Community health professor from the Jordan University of Science and Technology (1)

- Syrian refugee patients (one male and 1 female)

- International Rescue Committee staff (7) Including community health volunteers (CHVs) (one male, 1 female).

- Representatives from Médecins Sans Frontières, Medair, Altkaful, Royal Health Awareness Society, ABT Associates, International Medical Corps (6)

- Jordanian Ministry of Health (2)

- UNHCR (1)

Domains of semi-structured interviews

- Components of the Jordanian health system accessed by Syrian refugees.

- Linkages between elements of the health system.

- Barriers and facilitators in accessing care for diabetes and hypertension (HTN).

- Current role of CHVs.

- Potential roles of CHVs in care of patients with diabetes and HTN.

- Opportunities and constraints in realising the potential of CHVs in managing diabetes and high blood pressure. 


\section{Box 2 Stakeholder workshop proceedings}

\section{Day 1}

- Review workshop objectives.

- Review regional data on NCDs, community health volunteer (CHV) programming.

- Stakeholder training on causal loop diagrams (CLDs).

- Mixed stakeholder small groups generate their own CLDs.

- Present results of research team analyses-preliminary CLD and discussion of all variables.

\section{Day 2}

- Mixed stakeholder small groups comment and edit preliminary CLD.

- Whole stakeholder group discussion on preliminary CLD, with realtime refinement.

- Final CLD and variables generated (boxes 2 and 3).

- Stakeholder group identifies barriers and opportunities within the system to improve health using $\mathrm{CHV}$ strategies.

Jordan and were aware of the motivations and goals for this work. Community perspectives as well as the perspectives of CHVs who had practised in target communities, often for years, were prioritised in order to fully consider the limitations of current programming and the ways in which future programming might meet identified gaps in care. Individuals were interviewed by the research team using a semi-structured interview guide (box 1). All interviews were carried out in the respondent's preferred language, English or Arabic, in private locations outside of the home. Participants were contacted by a combination of phone, email and in-person requests to participate. All approached agreed to participate; extensive notes were taken during each roughly 1-hour interview. To ensure the privacy of the respondents, no interviews were recorded.

PKP and RR used content analysis strategies to identify key themes from these interviews. ${ }^{24}$ These themes were used to generate variables for inclusion in a preliminary CLD as well as relationships between each of these variables. ${ }^{25}$ All CLDs were generated and edited using VensimPLE software. ${ }^{26}$

During a 2-day workshop held in Amman in June 2019, stakeholders discussed and refined this preliminary CLD, including all variables and definitions generated by the research team. A summary of workshop proceedings is included in box 2 . The objective was to identify gaps and strengths in the current model of provision of NCD care for Syrian refugees and then to identify CHV-led strategies to fill key gaps while building on strengths in the system and incorporating mitigation strategies for anticipated barriers. A broad group of stakeholders were invited to comment on all aspects of this complex system. Five male and female CHVs represented perspectives on current and potential roles for CHVs in Jordan and the ways in which their roles might be expanded to address unmet needs. Similarly, Syrian refugee patients shared perspectives on challenges faced. NGO, MoH and United Nations (UN)/WHO representatives provided programmatic and funding context, and IRC leadership provided perspectives based on their management of the current CHV programme. Workshop facilitators presented data from a community-based survey of IRC catchment areas conducted during March/April of 2019 examining prevalence of disease, medication adherence, self-reported prevalence of complications and access to care among beneficiaries with diabetes and/or HTN. ${ }^{3}$ Additionally, workshop facilitators presented a summary of a global literature review on the roles of CHVs in the management of NCDs. This was done in order to orient stakeholders less familiar with community health approaches and more familiar with primary care for NCDs, programming orientation and context.

Workshop participants first met in small, mixed stakeholder groups to generate their own CLDs focusing on the outcome of the health of Syrian refugees with diabetes and HTN. Each small group presented their work to the larger group for comparison and integration. The research team then presented the preliminary CLD to the group, who then collaboratively edited the preliminary diagram in small groups and later as a whole. This exercise led to the final collaboratively built CLD (figure 1). Then, the group commented on barriers and opportunities within this system to achieving a CHV strategy with capacity to improve diabetes and high blood pressure outcomes.

A final list of variables generated by the combination of research team content analysis of qualitative data and stakeholder feedback during the 2-day workshop is listed in box 3 . These variables and linkages are summarised in the final CLD in figure 1, which, using positive and negative signs, considered how various components interact to ultimately affect health status. The central outcome of interest, 'health of Syrian refugees with dDM/HTN', has been highlighted in the CLD in red. Means by which CHVs might support the health of Syrian refugees in the Jordanian context as identified by this mixed stakeholder workshop are presented as follows, along with barriers that may limit opportunities.

\section{Patient and public involvement}

Syrian patients and Syrian CHVs participated in the workshop to gather their perceptions and insight into the needs of patients with NCD. They were not directly involved in the development of the research design.

\section{RESULTS}

Variables are described as follows and arranged in the CLD according to relevance at a national/global policy level, health system level, community health level and patient level. Relationships between variables are described in the final CLD with arrows: two variables linked by an arrow and a positive $(+)$ sign indicates that when one variable is influenced positively or negatively, the other variable also increases or decreases in importance. Similarly, variables linked by an arrow and a negative (-) sign indicate that 

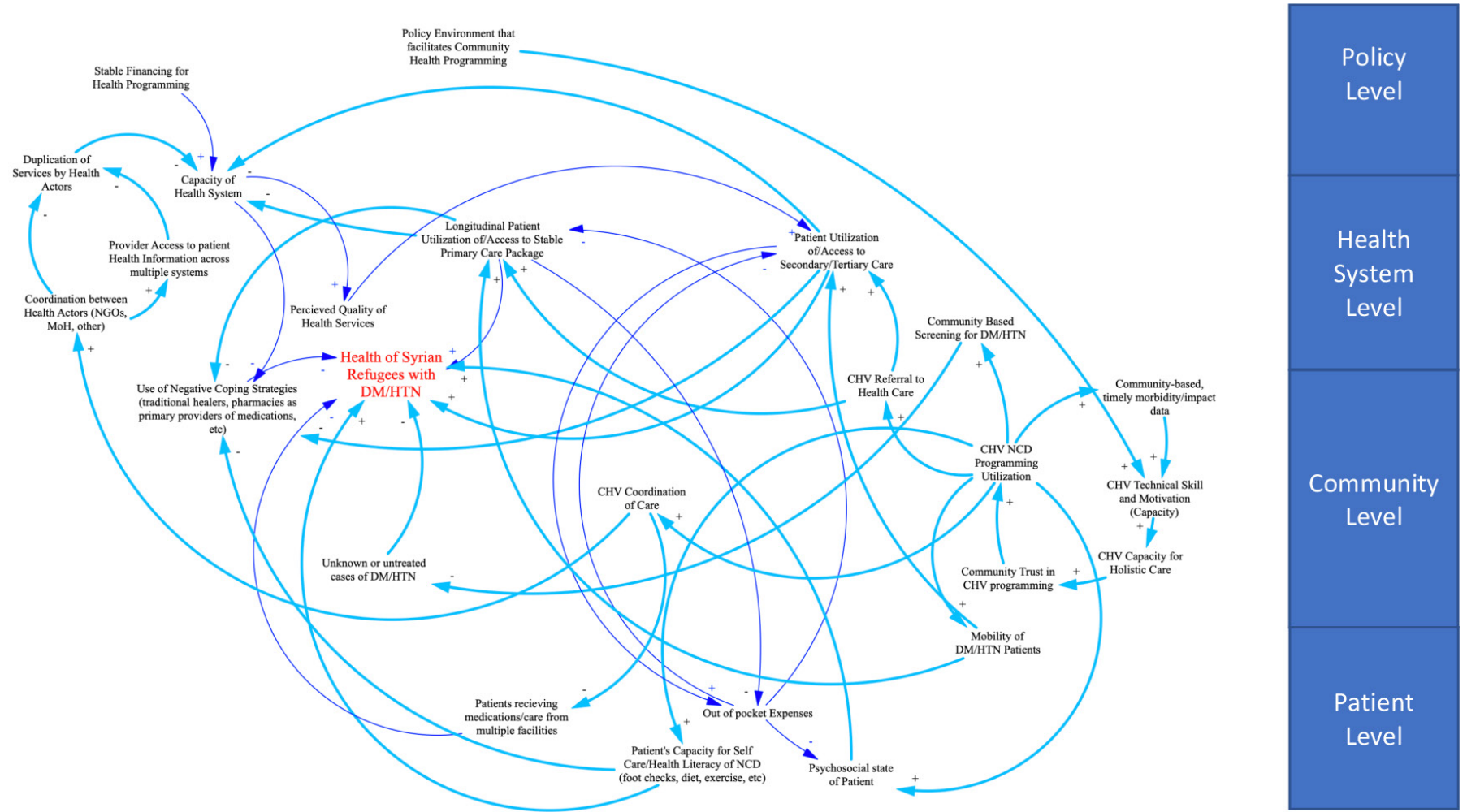

Figure 1 Final causal loop diagram: CHV programme for Syrian refugees and vulnerable Jordanians with DM/HTN. CHV, community health volunteer; DM, diabetes mellitus; HTN, hypertension; NCD, non-communicable disease.

when one variable varies, the linked variable goes in the opposite direction.

\section{Current challenges in NCD delivery and use}

The following section outlines current challenges in NCD delivery and use at each level of the health system. Linkages describing the existing system are highlighted by thin dark blue arrows in the final CLD (figure 1).

\section{Patient/community level}

Out-of-pocket expenses were identified as the single most important factor affecting health-seeking behaviour at the patient level (box 4, quotes 1 and 2). The patient's capacity for self-care and health literacy was deemed central to appropriate management of diabetes and HTN. This includes patients' understanding of the chronic nature of their disease and treatment, the need for daily medication, routine dietary and exercise needs, and avoidance of harmful behaviours such as smoking, as well as timely identification of complications. Patients receiving care from multiple facilities were seen as often suffering negative consequences as a result. It was recognised by stakeholders that patients may seek duplicate services as a relatively common coping mechanism, given limited and changing clinical services available to refugees. The psychosocial state of the patient was recognised as key in care-seeking behaviour, as refugees are predisposed to suffer from serious mental illness and trauma, which may introduce feelings of hopelessness and impair the ability to prioritise management of chronic health conditions. Finally, patients with impaired mobility, including women who must remain at home, the elderly and disabled, often have a challenging time travelling to health facilities (box 4, quote 3 ).

At the community level, stakeholders identified unknown/untreated cases of diabetes and HTN as a potentially large unmet need and knowledge gap. In the context of limited access to primary care, refugees face barriers to obtaining a diagnosis (including seeking care only when conditions become symptomatic) and a lack of exposure to information about NCDs. Finally, in an environment where services available to patients often change with shifting policy goals and funding priorities, patients often engage in negative coping strategies to fill gaps in care. These may involve seeking care from non-physician providers, including traditional healers who provide herbal remedies, seeking medications directly from pharmacies without the involvement of a physician, taking prescribed medications in lower doses or less frequently to make them last longer, or forgoing medications entirely despite a known diagnosis. The aforementioned patient factors, in particular high out-of-pocket expenses, patient's (most often suboptimal) capacity for self-care and health literacy, and impaired psychosocial state of the patient, as well as use of negative coping strategies, could potentially result in poor health outcomes.

Health system level

Stakeholders identified several factors at the level of the health system that affect the health of Syrian refugees. The capacity of the health system was identified as suffering 


\section{Box 3 Final list of variables}

\section{Patient/community}

- Psychosocial state of patient.

- Patient capacity for self-care and health literacy.

- Out-of-pocket expenses.

- Mobility of patients.

- Patients receiving care from multiple facilities.

- Unknown or untreated cases of diabates mellitus (DM)/hypertension (HTN).

Use of negative coping strategies.

\section{Health system}

- Capacity of health system.

- Longitudinal patient access/Utilization of primary care.

- Patient utilization of/access to secondary/tertiary care.

- Coordination of between health actors.

- Provider access to health information across systems.

- Perceived quality of health services.

\section{Policy}

- Stable financing for health programming.

Duplication of services by health actors.

- Policy environment that facilitates community health programming.

Roles of community health volunteers (CHVs)

- CHV program use.

- Community-based, timely morbidity/impact data.

- CHV technical skill and motivation.

- CHV capacity for holistic care.

- Community trust in CHVs.

- Community-based screening for DM/HTN.

- CHV coordination of care.

CHV referral to care.

negatively from a lack of coordination between health actors ( $\mathrm{NGOs}$ and $\mathrm{MoH}$ ). Stakeholders, particularly medical providers, felt that a lack of provider access to health information across multiple systems was responsible, at least in part, for avoidable duplication of services. Stakeholders described unknowingly repeating testing or providing multiple prescriptions for either duplicate or potentially interacting medications to patients who receive care from multiple health centres (box 4, quote 4). This was not only flagged as a danger to patients given challenges monitoring those who seek care from multiple providers, it was also seen as a strain on limited resources when services are duplicated. Generally, key informants and workshop participants agreed that $\mathrm{MoH}$ services were strained, and many patients, CHVs and health actors perceived the quality of $\mathrm{MoH}$ services to have suffered as a result. Improved coordination between health NGOs had, per participants, mitigated many of these issues during the response; however, most felt coordination could be improved, particularly with regard to access to medical records across health systems (via patient-held records or formal mechanisms for sharing information). Stakeholders uniformly felt that the capacity of the health system was harmed by a lack of stable financing for health programming.

\section{Box 4 Quotes from key informant interviews}

1. Previously there were many NGOs providing services, but now there are less; there are just a few that provide free services. [Humanitarian NGO] can provide some services, but they charge and even this subsidized rate is challenging. Only [different humanitarian NGO] is providing free services, and said [third humanitarian NGO] is not taking patients. If services or medications are not available in these places, I have to buy it externally. - Syrian refugee

2. Finances are the main barrier. Even if you give money to an old man with diabetes, if he has five children he will feed them and prioritize rent over his own health. Rental costs are very high and this is challenging. When we do our post-cash-transfer calls, we ask why are you still in debt? Recipients will often reply that they had to pay rent to avoid becoming homeless. - Non-governmental organisation (NGO) health programme administrator

3. When we went to the field, some women had instructions from husbands that they could not go outside their home. They when they saw the CHVs, said, "thank God! If you can help me here in the home that is much better". - International Rescue Committee community health volunteer (CHV)

4. The only link between different health providers is the patient. We try to get records of investigations from other NGOS, etc-but most patients come in with no records, no data, no labs, no access to files from MoH or other NGOs. Strict privacy policies prevent coordination. This gets in the way of patient care. - NGO health provider

5. NCDs need time-intensive care, at least $20-30$ min per visit, but health providers don't have that time. Most providers focus on treatment and not prevention - and health messages need to be repeated many times. There aren't adequate human resources for this. Health providers focus on treatment, not prevention. - NGO health programme administrator

6. When I started, we were attending NCD working group health sector meetings. I noted that these are not run as efficiently as they should be. It was a parade of bragging. No one is collaborating and actually sorting out what they should be doing, NGOs fight to maximize funding. There seems to be no entity regulating/coordinating which organization should focus on what specific issues, etc. — NGO health programme administrator

7. If a CHV calls and states that there are 70 cases of diarrhea in one community, with dehydration in this informal tent settlement, then we can go there. If well trained, these CHVs can provide vital information in real time. They can do clinical assessments as well if trained well. You don't need health care professionals to measure blood pressure or blood glucose, CHVs can do this. CHVs can save time/cost and prevent complications. They can identify complication early. If there is a certain case that needs a referral, then they can refer. I tell my CHVs, "You are all soldiers". CHVs save people's lives. - CHV programme administrator

8. Will there be real action, or just talking? - Syrian patient, male

Barriers to longitudinal access to and utilization of primary care, largely resulting from the patient/community factors described earlier, were felt to substantially impact health outcomes among refugees with NCDs. Participants highlighted that geographical and financial access might not always automatically result in medically necessary, longitudinal utilization by patients, with factors such as health literacy and competing household priorities playing an important role (box 4, quote 2). Access to and utilization of secondary and tertiary care, when needed, were recognised 
as critical to the health of patients with diabetes and HTN - particularly those who had experienced a complication of their disease. It was also recognised, both in KIIs and the workshop, that humanitarian agencies largely focus on primary care, with refugees facing very limited and typically expensive access to secondary or tertiary care (box 4, quote 5).

\section{Policy level}

Stakeholders identified key policy level variables related to health status. Typical funding cycles for humanitarian programmes are in the range of 1-2years, while NCD programming was felt to require a longer-term strategy. Donor funding of humanitarian NGO health programming has also decreased in the region per workshop participants, which has resulted in a lack of the type of stable financing for health programming that is critical for longitudinal care of diabetes and HTN (box 4, quote 1). Workshop participants noted that the $\mathrm{MoH}$ was in the challenging position of having to provide for a large influx of refugees with limited funding, which has resulted in changing policies with regard to fees charged to Syrian refugees for health services. These changing policies have, in themselves, resulted in distrust and inaccessibility among refugees with regard to $\mathrm{MoH}$ health services. Duplication of services by health actors in the domain of NCD care, with multiple providers present in some geographical areas with relatively fewer in others, was felt by participants to have resulted in inefficient use of limited funding. Several key informants cited a lack of coordination of humanitarian programming nationally, resulting in overlapping programming in some locations and a lack of services in other geographical regions (box 4, quote 6).

\section{Potential for CHV programming}

Stakeholders were asked to consider how a CHV programme might, both positively and negatively, impact the health of Syrian refugees within the systems described previously. Linkages representing areas of potential impact are highlighted with thick light blue arrows in the CLD (figure 1).

CHV capacity, including their technical skills, workload, appropriate remuneration and motivation, were seen as central to the success of any CHV programme. CHVs draw from the communities they serve-thus, their understanding of the social and cultural context was also seen as a tremendous asset to their work. Current policy in Jordan allows for limited payment of CHVs, which serves as a barrier. They have to been seen as highly skilled and reliable by the communities they serve, reflected in the variable community trust in CHV programming. CHV capacity for holistic care, namely, the ability to make accurate and timely referrals to resources not related to the central goals of their programme (food aid, services for survivors of violence, psychosocial aid and cash transfers), was considered central to securing patient engagement. Stakeholders, including CHVs themselves, agreed that each time a CHV is unable to meet the need of a patient whether or not it is related to healthcare, that patient loses trust in CHV services. As a result, that patient may not engage with CHVs on health-related messaging and services in the future. However, CHVs stated that timely and meaningful referrals require improved coordination between humanitarian actors to ensure that CHVs do not make referrals to services that are no longer available. CHV capacity, community trust in CHV programming and CHV capacity for holistic care were thought to positively reinforce CHV NCD programme utilization by the target beneficiaries.

Stakeholders identified multiple ways that a CHV NCD programme might support the health of patients at multiple levels beyond the current system. CHVs were felt to be a critical conduit for increased patient knowledge on management of their disease, specifically increasing patient's capacity for self-care and health literacy. CHVs could strengthen health literacy and self-management by providing targeted health messaging (on foot checks, adherence to medication and clinic visits) (box 4, quote 7). CHVs might also serve as a means of screening adults to identify undiagnosed cases of diabetes and HTN, provide community-based, timely morbidity/impact data, which might strengthen access to diagnoses and care as well as target particularly high-risk patients. CHVs could also provide basic psychosocial support and referrals to more advanced care for patients suffering from more acute mental health needs. CHVs were also felt to be an important means of combating negative coping strategies, both via education and referrals to services that might allow for more stable access to medications and allopathic primary care. Stakeholders identified patients with limited mobility as a key population that might greatly benefit from CHVs (box 4, quote 3). CHVs could, with training, provide homebased blood glucose and blood pressure measurement, and coordinate with clinics to provide disease management and health education, reducing the need for more frequent visits to a health facility.

Though CHV led, community-based screening might increase the burden on the health system, stakeholders felt that a robust CHV programme might also decrease the burden on fixed clinics by reducing duplication of services. CHVs could do this by helping patients coordinate care within one health system as much as possible and aligning services between multiple health providers when needed. CHVs might also allow health facilities to focus more on medical management during appointments by delivering health education in patient households. National policies prevent those who are not licensed nurses or physicians from measuring blood glucose and blood pressure as a part of the provision of healthcare, though measurement devices are available to households for purchase and private use. This and the need for consistent funding sources highlight the importance of a policy environment that facilitates community health programming.

Stakeholders felt that increased use of primary care and preparedness for routine NCD management of some complications at the PHC level might decrease 
the reliance on scarce secondary and tertiary resources. However, participants noted that such an increase in primary care use would create a strain on the capacity of the health system as it risks inadequate resources and planning. Overall capacity of the health system was thought to be an essential component of high-quality primary, secondary and tertiary care.

\section{DISCUSSION}

This causal loop analysis workshop identified many issues borne out by previous studies among Syrian refugees in the region, highlighting costs, challenges with changing capacities and services of humanitarian actors, lack of coordination between humanitarian actors as well as the $\mathrm{MoH}$, shifting national policies regarding provision of healthcare by state facilities to refugees, and very limited access to secondary and tertiary care as major barriers in assuring continuous care. ${ }^{11122327}$ Based on the results of this causal loop analysis workshop, the research team and programme staff identified multiple CHV strategies that align with an evidence-based conceptual model of care for priority NCDs through primary care systems developed by Kane $e t a l^{28}$ These strategies and relevant evidence are presented as follows.

\section{CHV-led community-based prevention via risk factor modification}

A CHV-led community-based prevention programme focusing on risk factor modification would enable CHVs to provide targeted health education messaging to refugee communities on primary prevention, including smoking, diet and exercise, through tailored discussion of realistic goals given context-specific constraints. CHVs should engage the entire household on risk factor modification. ${ }^{49}$ There are, however, complex issues with regard to diet (e.g., food insecurity), smoking cessation (e.g., high prevalence and culture) and promoting exercise (e.g., few options for female heads of households) among Syrian refugees. ${ }^{4529} 30$

\section{CHV screening of Syrian refugee communities for diabetes and HTN}

Diabetes and HTN may remain undiagnosed among Syrian refugees in Jordan. ${ }^{3}$ However, evidence suggests that screening in the community using random blood glucose and blood pressure has low specificity and may yield excessive case numbers that, when not appropriately discussed with a physician, result in limited linkage to care, and may result in low compliance with treatment and unclear impact on health. ${ }^{15}{ }^{31}$ In a population-based screening in Saudi Arabia, only a limited proportion of those who screened positive for disease were appropriately linked to care. ${ }^{32}$ Additionally, generating high levels of demand that cannot be appropriately met may not be ethical. ${ }^{9}$ Thus, CHV-led screening might target patient household members $\geq 30$ years, those with a history of gestational diabetes and HTN or those who report symptoms that might be consistent with complications of an NCD during the course of a CHV household visit.

\section{CHV support of adherence to medication, disease management and clinical care}

Refugees may have limited understanding of how to best manage NCDs and avoid complications, given little interaction with healthcare. ${ }^{45}$ Studies of CHV interventions in rural Iran, Nepal and Mexico have shown that CHV programming designed to improve adherence to care and to identify complications early can improve patient outcomes in non-refugee settings. ${ }^{151633}$ Those with the poorest control tend to benefit the most from this type of CHV intervention. ${ }^{16}$ In light of the relatively high burden of NCDs in many humanitarian contexts and limited resources, CHV interventions that focus on the most poorly compliant or controlled patients may have the highest impact. Strategies outlined in the WHO's Package of Essential NCD Interventions and tools created by the International Federation of the Red Cross and Red Crescent can be carefully adapted for CHV interventions, including out-of-clinic biological monitoring to better identify patients in need of urgent or emergent referrals to clinical care; identification criteria for emergent and urgent referral of complications via simple case definitions for presentations of diabetic foot ulcers, hyperglycaemia or hypoglycaemia, myocardial infarction and unstable angina; and modules for self-management counselling and psychosocial support. ${ }^{30} 3435$ Importantly, the CHV would provide patients with preliminary identification of a suspect condition or complications followed by a direct referral to primary or secondary care services.

\section{CHV provision of mental health and psychosocial support}

Conflict and displacement-affected patients may face mental illness barriers in dealing with NCDs. This may, in turn, affect compliance, adherence and motivation and result in increased reliance on negative coping mechanisms. ${ }^{12}{ }^{17}$ Stigma often prevents many Syrian refugees from seeking care for psychiatric needs, and clinic-based psychological services often do not have the capacity to meet the needs of these patients. There is some evidence of the effectiveness of psychosocial support for HIV adherence and better evidence for the application of mental health and psychosocial services in the context of a CHV programme. ${ }^{36}$ Thus, a CHV NCD-focused programme should address the identification of psychosocial health needs in some form, with referral to mental health services. ${ }^{30} 3738$

\section{Standardised referral pathways (from clinic) and follow-up (to clinic)}

Patients face multiple challenges in engaging in primary care longitudinally, as described previously. ${ }^{12}$ Evidence suggests strategies that might support improved patient adherence to primary care. SMS-based reminders sent to Syrian refugees with NCDs in Lebanon improved patient attendance to clinic appointments. ${ }^{39} \mathrm{~A}$ patient-held 
medical record trialled in Chiapas, Mexico, contributed to a CHV intervention to improve patient adherence to care and patient understanding of their own health needs. ${ }^{16}$ With regard to secondary and tertiary care, referral pathways are complex and result in significant delays in care, and care itself is often expensive. ${ }^{40}$ Secondary care options are, in most humanitarian contexts, insufficient to meet needs. Additionally, limited coordination between clinical providers (NGOs, $\mathrm{MoH}$ and private) hinders referrals and data sharing. ${ }^{5} \mathrm{CHV}$ s may play an important role in coordinating care for Syrian refugees with NCDs in Jordan. ${ }^{1629}$

\section{Limitations}

Workshop facilitators relied on Arabic translation during the exercise, as both Arabic and English speakers participated. A diverse set of stakeholders, including Syrian refugees, vulnerable Jordanians, CHVs, physicians and health programmes, as well as national/UN leadership, were invited. While every effort was made to encourage full participation, it is possible that in this environment, some community members and CHVs felt unable to fully participate. Interviews were conducted by an Englishspeaking researcher with the assistance of an Arabicspeaking researcher as needed, which may have resulted in interviewee discomfort or subtle translation errors.

\section{CONCLUSION}

Syrian refugees with diabetes and HTN face multiple barriers in accessing care, within a complex system. CHVs potentially provide an efficient means of helping this vulnerable population navigate this system. Further studies on the effectiveness of CHV interventions on NCDs among Syrian refugees in Jordan are needed.

\section{Author affiliations}

${ }^{1}$ Department of Emergency Medicine, University of Southern California, Los Angeles, California, USA

${ }^{2}$ International Rescue Committee, Amman, Jordan

${ }^{3}$ Department of Community and Mental Health Nursing, Jordan University of Science and Technology, Irbid, Jordan

${ }^{4}$ International Rescue Committee, New York, New York, USA

${ }^{5}$ Infectious Disease Epidemiology, London School of Hygiene and Tropical Medicine, London, UK

Contributors PKP and RR designed the study; NA-A, RAAR, MF, KAA and RA-M provided critical support during the causal loop analysis workshop; PKP, RR and FR analysed and interpreted the data. PKP and RR wrote the article. All authors made critical revisions to the article and read and approved the final version of the manuscript.

Funding This work was supported by Enhancing Learning \& Research for Humanitarian Assistance, Research for Health in Humanitarian Crises (grant number 28368).

\section{Competing interests None declared.}

Patient and public involvement Patients and/or the public were not involved in the design, conduct, reporting or dissemination plans of this research.

Patient consent for publication Not required.

Ethics approval This study was approved by the institutional review boards (IRBs) of the University of Southern California (\#HS-18--00569, H 1.00.020), the International Rescue Committee (IRB \#: 00009752 FWA \#: 00022773) and Jordan
University of Science and Technology (83/117/2018, 57/28/2018). Key informants gave written consent to participate and workshop participants gave verbal consent. Individuals were asked to keep the contents of the discussions during the workshops confidential. The contents of key informant interviews were summarised and shared in the workshop in a deidentified manner.

Provenance and peer review Not commissioned; externally peer reviewed.

Data availability statement No data are available. The qualitative data from this study will not be made publicly available due to the ease by which interviewees may be identified through the full transcripts, even if key sections are redacted. Furthermore, during the informed consent process, the participants did not consent to make the data publicly available.

Open access This is an open access article distributed in accordance with the Creative Commons Attribution Non Commercial (CC BY-NC 4.0) license, which permits others to distribute, remix, adapt, build upon this work non-commercially, and license their derivative works on different terms, provided the original work is properly cited, appropriate credit is given, any changes made indicated, and the use is non-commercial. See: http://creativecommons.org/licenses/by-nc/4.0/.

\section{ORCID iDs}

Parveen K Parmar http://orcid.org/0000-0002-2447-6690

Ruwan Ratnayake http://orcid.org/0000-0003-4978-6668

\section{REFERENCES}

1 Operational portal refugee situations. Situation Syria regional refugee response, 2020. Available: https://data2.unhcr.org/en/situations/syria

2 Rehr M, Shoaib M, Ellithy S, et al. Prevalence of non-communicable diseases and access to care among non-cAMP Syrian refugees in northern Jordan. Confl Health 2018;12:33.

3 Ratnayake R, Rawashdeh F, AbuAIRub R, et al. Access to care and prevalence of hypertension and diabetes among Syrian refugees in northern Jordan. JAMA Netw Open 2020;3:e2021678.

4 Elliott JA, Das D, Cavailler P, et al. A cross-sectional assessment of diabetes self-management, education and support needs of Syrian refugee patients living with diabetes in Bekaa Valley Lebanon. Confl Health 2018;12.

5 Boulle P, Kehlenbrink S, Smith J, et al. Challenges associated with providing diabetes care in humanitarian settings. Lancet Diabetes Endocrinol 2019;7:648-56.

6 Spiegel PB, Checchi F, Colombo S, et al. Health-Care needs of people affected by conflict: future trends and changing frameworks. The Lancet 2010;375:341-5.

7 É A, Maconick L, Ellithy S. "To die is better for me" social suffering among Syrian refugees at a noncommunicable disease clinic in Jordan: a qualitative study. Confl Health 2019;14:1-31.

8 Ansbro Éimhín, Garry S, Karir V, et al. Delivering a primary-level noncommunicable disease programme for Syrian refugees and the host population in Jordan: a descriptive costing study. Health Policy Plan 2020;35:931-40.

9 Slama S, Kim H-J, Roglic G, et al. Care of non-communicable diseases in emergencies. Lancet 2017;389:326-30.

10 World Health Organization Eastern Mediterranean Region. Health of refugees and migrants practices in addressing the health needs of refugees and migrants WHO Eastern Mediterranean region 2018 2018

11 Doocy S, Lyles E, Akhu-Zaheya L, et al. Health service utilization among Syrian refugees with chronic health conditions in Jordan. PLoS One 2016;11:e0150088-12.

12 Zahrah Mcnatt Z, Freels PE, Chandler H. What's happening in Syria even affects the rocks": a qualitative study of the Syrian refugee experience accessing noncommunicable disease services in Jordan. Confl Health 2019;13:1-6.

13 Washington $\mathrm{CH}$, Tyler FJ, Davis J, et al. Trauma training course: innovative teaching models and methods for training health workers in active conflict zones of eastern Myanmar. Int J Emerg Med 2014;7.

14 Musoke D, Ndejjo R, Kiracho EE. Health for the people: national community health worker programs from Afghanistan to Zimbabwe 2020.

15 Farzadfar F, Murray CJL, Gakidou E, et al. Effectiveness of diabetes and hypertension management by rural primary health-care workers (Behvarz workers) in Iran: a nationally representative observational study. The Lancet 2012;379:47-54.

16 Newman PM, Franke MF, Arrieta J, et al. Community health workers improve disease control and medication adherence among patients with diabetes and/or hypertension in Chiapas, 
Mexico: an observational stepped-wedge study. BMJ Glob Health 2018;3:e000566.

17 Neupane D, McLachlan CS, Mishra SR, et al. Effectiveness of a lifestyle intervention led by female community health volunteers versus usual care in blood pressure reduction (COBIN): an open-label, cluster-randomised trial. The Lancet Global Health 2018;6:e66-73.

18 Sub Centre H. Module for multi-purpose workers (MPW)-female/male on prevention, screening and control of common non-communicable diseases.

19 Varghese J, Kutty VR, Paina L, et al. Advancing the application of systems thinking in health: understanding the growing complexity governing immunization services in Kerala, India. Health Res Policy Syst 2014;12:47.

$20 \mathrm{Li} \mathrm{Q}$, Hyder AA, Peters DH. Advancing the application of systems thinking in health: why cure crowds out prevention. Heal Res Policy Syst 2014;12:1-5.

21 Paina L, Bennett S, Ssengooba F, et al. Advancing the application of systems thinking in health: exploring dual practice and its management in Kampala, Uganda. Health Res Policy Syst 2014;12:41.

22 Williams B, Hummelbrunner R. Systems concepts in action. A practical toolkit. Stanford, California: Stanford University Press, 2011.

23 Zablith N, Diaconu K, Naja F, et al. Dynamics of non-communicable disease prevention, diagnosis and control in Lebanon, a fragile setting. Confl Health 2021;15:4.

24 Hsieh H-F, Shannon SE. Three approaches to qualitative content analysis. Qual Health Res 2005;15:1277-88.

25 Elo S, Kyngäs $\mathrm{H}$. The qualitative content analysis process. J Adv Nurs 2008;62:107-15.

26 Vensim PLE. Vensim® personal learning edition, 2020. Available: https://vensim.com/vensim-personal-learning-edition/

27 Akik C, Ghattas H, Mesmar S, et al. Host country responses to noncommunicable diseases amongst Syrian refugees: a review. Confl Health 2019;13.

28 Kane J, Landes M, Carroll C, et al. A systematic review of primary care models for non-communicable disease interventions in subSaharan Africa. BMC Fam Pract 2017;18:1-12.
29 Sethi S, Jonsson R, Skaff R, et al. Community-Based noncommunicable disease care for Syrian refugees in Lebanon. Glob Health Sci Pract 2017;5:495-506.

30 International Federatin of the Red Cross. Healthy lifestyle toolkit IFRC, 2016. Available: https://www.ifrc.org/en/what-we-do/health/ diseases/noncommunicable-diseases/ncds-toolkit/

31 American Diabetic Association. Screening for type 2 diabetes, 2003. Available: https://care.diabetesjournals.org/content/diacare/26/ suppl_1/s21.full.pdf

32 Al-Ghamdi AJ, Al-Turki KA, Al-Baghli NA, et al. A community-based screening campaign for the detection of diabetes mellitus and hypertension in the eastern Province, Saudi Arabia: methods and participation rates. J Family Community Med 2007;14:91-7.

33 Abdel-All M, Thrift AG, Riddell M, et al. Evaluation of a training program of hypertension for accredited social health activists (ASHA) in rural India. BMC Health Serv Res 2018;18:320.

34 Mendis S. Package of essential NCD interventions for primary health care in humanitarian emergencies $(\mathrm{PEN}-\mathrm{H}) 2019$

35 Kehlenbrink S, Smith J, É A. The burden of diabetes and use of diabetes care in humanitarian crises in low-income and middleincome countries. Lancet Diabetes Endocrinol 2019;8587:1-10.

36 Han H-R, Kim K, Murphy J, et al. Community health worker interventions to promote psychosocial outcomes among people living with HIV-A systematic review. PLoS One 2018;13:e0194928.

37 Ommeren MV, Hanna F, Weissbecker I, et al. Mental health and psychosocial support in humanitarian emergencies. East Mediterr Health J 2015;12:498-502.

38 Kayali M, Moussally K, Lakis C, et al. Treating Syrian refugees with diabetes and hypertension in Shatila refugee cAMP, Lebanon: Médecins sans Frontières model of care and treatment outcomes. Confl Health 2019;13:1-11.

39 Doocy S, Paik KE, Lyles E, et al. Guidelines and mHealth to improve quality of hypertension and type 2 diabetes care for vulnerable populations in Lebanon: longitudinal cohort study. JMIR Mhealth Uhealth 2017;5:e158.

40 Akik C, Ghattas H, Mesmar S, et al. Host country responses to noncommunicable diseases amongst Syrian refugees: a review. Confl Health 2019;13:8. 